International Journal of Pure and Applied Mathematics

Volume 85 No. 2 2013, 203-210

ISSN: 1311-8080 (printed version); ISSN: 1314-3395 (on-line version)

url: http://www.ijpam.eu

doi: http://dx.doi.org/10.12732/ijpam.v85i2.2

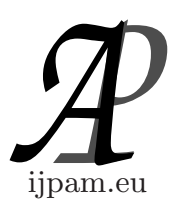

\title{
AN EFFICIENT NUMERICAL METHOD FOR SOLVING FREDHOLM INTEGRAL EQUATIONS OVER $(0,+\infty)$
}

\author{
M.T. Darvishi ${ }^{1}$, A. Omidi ${ }^{2}$, F. Khani ${ }^{3} \S$ \\ $1,2,3$ Department of Mathematics \\ Razi University \\ Kermanshah, IRAN
}

\begin{abstract}
In this paper, two types of integral equations of the second kind over semiaxis are presented. Then we introduce a numerical method by using the product rule and Nyström method. The method reduces the integral equation to a system of linear algebraic equations. Some numerical examples are presented to show the efficiency and accuracy of the method.
\end{abstract}

AMS Subject Classification: 45B05, 45G10

Key Words: extended Lagrange interpolation, product rule, Nyström method

\section{Introduction}

Let us consider the following Fredholm integral equation

$$
f(y)-\int_{0}^{+\infty} k(x, y) f(x) u(x) d x=g(y), \quad y \in(0,+\infty),
$$

where $k(x, y)$ and $g(y)$ are known functions, $u(x)=(1+x)^{\lambda} x^{\gamma} e^{\frac{-x^{\beta}}{2}}$, is a generalized Laguerre weight, where $x \in \mathbb{R}^{+}, \alpha>-1, \beta>\frac{1}{2}, \gamma \geq-1, \lambda \geq 0$ and $f$ is the unknown function which should be determined.

Received: September 19, 2012

(C) 2013 Academic Publications, Ltd.

$\S$ Correspondence author url: www.acadpubl.eu 


\section{Preliminaries}

\subsection{Functional Spaces}

In the sequel we consider the following set of continuous functions on $\mathbb{R}^{+}=$ $(0,+\infty),\left(f \in C^{0}\left(\mathbb{R}^{+}\right)\right)$and

$$
C_{u}:=\left\{f \in C^{0}\left(\mathbb{R}^{+}\right): \lim _{x \rightarrow 0^{+}} f(x) u(x)=0=\lim _{x \rightarrow+\infty} f(x) u(x)\right\}
$$

with the norm

$$
\|f\|_{C_{u}}:=\|f u\|:=\max _{x \geq 0}|(f u)(x)| .
$$

Moreover, we denote by $W_{r}=W_{r}(u), \quad r \geq 1$, the Sobolev-type space defined by

$$
W_{r}=W_{r}(u)=\left\{f \in C_{u}: f^{(r-1)} \in A C\left(\mathbb{R}^{+}\right) \text {and }\left\|f^{(r)} \varphi^{r} u\right\|<+\infty\right\},
$$

with the norm

$$
\|f\|_{w_{r}}:=\|f u\|+\left\|f^{(r)} \varphi^{r} u\right\|
$$

(see [8] and [5]) $A C$ stand for absolutly continuous.

\subsection{Extended Lagrange Interpolation}

We denote by $\mathcal{P}_{n}$ the set of all polynomials of degree at most $n$. For sufficiently large $n$ (say $n>n_{0}$ ) we introduce the quantity $a_{n}=a_{n}(w)=4 \frac{\Gamma(\beta)^{\frac{2}{\beta}}}{\Gamma(2 \beta)^{\frac{1}{\beta}}} m^{\frac{1}{\beta}}$ (Maskhar-Rachmanove-saff numbers, see [7]). Here after $C$ denotes a positive constant which may be different in different formulas. We write $C \neq C(a, b, \ldots)$ to say that $C$ is a constant independent of the parameters $a, b, \ldots$ and we write $C=C(a, b, \ldots)$ to say that $C$ depends on $a, b, \ldots$ Let $x_{k}=x_{n, k}, k=1,2, \ldots, n$ be zeros of the $n$-th Laguerre orthogonal polynomial ordered in increasing order. We recall that all of these zeros are contained in the interval $(0,4 m)$ and that $x_{m} \sim 4 m-m^{\frac{1}{3}}$ (see [9]). Let $w(x)=x^{\alpha} e^{-x^{\beta}}, \alpha>-1, \beta>\frac{1}{2}$. The polynomial $L_{n+1}^{*}(w, f ; x)$ denotes the Lagrange polynomial which interpolates a given function at the zeros of $p_{n}(w)$ and at $a_{n}$, that is

$$
L_{n+1}^{*}\left(w, f ; x_{k}\right)=\sum_{k=1}^{n+1} l_{k}^{*}(x) f\left(x_{k}\right), \quad x_{n+1}=a_{n},
$$


where

$$
l_{k}^{*}(x)=\frac{a_{n}-x}{a_{n}-x_{k}} \frac{p_{n}(w ; x)}{p_{n}^{\prime}\left(w ; x_{k}\right)\left(x-x_{k}\right)}, \quad k \leq n, \quad l_{n+1}^{*}(x)=\frac{p_{n}(w ; x)}{p_{n}\left(w ; a_{n}\right)} .
$$

For any fixed $0<\theta<1$, define

$$
x_{j}=x_{j}(w)=\min \left\{x_{k}: x_{k} \geq \theta a_{n}, \quad k=1,2, \ldots, n\right\} .
$$

We define

$$
L_{n+1}^{* *}(w, f ; x)=\sum_{k=1}^{j} l_{k}^{*}(x) f\left(x_{k}\right),
$$

the polynomial $L_{n+1}^{* *}(w, f ; x)$ belongs to a subspace of $\mathcal{P}_{n}$, namely $\mathcal{P}_{n}^{*}$ with

$$
\mathcal{P}_{n}^{*}=\left\{p \in \mathcal{P}_{n}: p\left(x_{i}\right)=p\left(a_{n}\right)=0, x_{i}>x_{j}\right\} \subset \mathcal{P}_{n}
$$

In other words, $L_{n+1}^{* *}: C_{u} \rightarrow \mathcal{P}_{n}^{*}$ is a projection from $C_{u}$ onto $\mathcal{P}_{n}^{*}$ (see [5] and $[8])$.

\subsection{Product Rule}

A Gaussian-type formula is the following so called "product rule"

$$
\begin{aligned}
\int_{0}^{+\infty} f(x) k(x, y) u(x) d x=\sum_{k=1}^{j} f\left(x_{k}\right) \lambda_{k}(y)+e_{n}^{*}(f, y) & \\
=: & I_{n}(f, y)+e_{n}^{*}(f, y),
\end{aligned}
$$

where $k:[0,+\infty) \times[0,+\infty) \rightarrow \mathbb{R}, \lambda_{k}(y)=\int_{0}^{+\infty} l_{k}^{*}(x) k(x, y) u(x) d x$, and $e_{m}^{*}(f)$ is the error of the quadrature rule [3].

\section{Numerical Results}

Now we return to the equation

$$
f(y)-\int_{0}^{+\infty} k(x, y) f(x) u(x) d x=g(y), \quad y \in(0,+\infty),
$$

we rewrite this equation in the operator form

$$
(I-\mathcal{K}) f=g
$$


where $I$ is the identity symbol. By multiplying both sides of the equation by the weight function $u(y)$, we have

$$
(f u)(y)-u(y) \int_{0}^{+\infty} k(x, y) f(x) u(x) d x=(g u)(y), \quad y \in[0,+\infty),
$$

approximating the integral in (4) by product rule, we get

$$
(f u)(y)-u(y) \sum_{k=1}^{j} f\left(x_{k}\right) \lambda_{k}(y)=(g u)(y)
$$

where

$$
\lambda_{k}(y)=\int_{0}^{+\infty} l_{k}^{*}(x) k(x, y) u(x) d x .
$$

Now, we apply the Nyström method, and get

$$
\sum_{k=1}^{j}\left[\delta_{i k}-\frac{u\left(x_{i}\right)}{u\left(x_{k}\right)} \lambda_{k}\left(x_{i}\right)\right] \alpha_{k}=(g u)\left(x_{i}\right), \quad i=1, \ldots, j
$$

from (5), we obtain $f\left(x_{k}\right)$ for $k=1, \ldots, j$. Then the approximate solution obtains by $\bar{f}_{n}(y)=\sum_{i=1}^{j} \varphi_{i}(y) \alpha_{i} \in \mathcal{P}_{n}^{*}$ with $\varphi_{i}(z)=\frac{l_{i}^{*}(z)}{u\left(x_{i}\right)}$ and $\alpha_{i}=f u\left(x_{i}\right)$.

\section{Numerical Solution of Another Kind of Fredholm Integral Equation}

In this section, we consider the integral equation of the form

$$
f(y)-\int_{0}^{+\infty} k(x, y) f(x) w(x) d x=g(y)
$$

where $w(x)=x^{\alpha} e^{-x}, \alpha>-1$ and $u(x)=\sqrt{w(x)}\left(\frac{x}{1+x}\right)^{a}(1+x)^{b}$. Moreover, let the real constants $a, b$ satisfy the conditions $a>-1+\max \left(\frac{-\alpha}{2}, \frac{1}{4}\right), b<\frac{-1}{4}$. We now offer a way to solve the equation (6). First, we introduce the following product rule

$$
\int_{0}^{+\infty} k(x, y) f(x) w(x) d x=\sum_{i=1}^{n} \lambda_{i}(y) f\left(x_{i}\right)+e_{n}^{*}(f, y)
$$


with

$$
\lambda_{i}(y)=\int_{0}^{+\infty} k(x, y) l_{i}(x) w(x) d x
$$

(see [9]). Now, we are going to represent a method which is based upon the product rule and the Nyström method for solving this kind of integral equation. To do this, we multiply both sides of equation (6) by $u(y)$, then by applying product rule we have

$$
(f u)(y)-u(y) \sum_{k=1}^{j} f\left(x_{k}\right) \lambda_{k}(y)=(g u)(y) .
$$

Now, we can apply the Nyström method, and get

$$
\sum_{k=1}^{j}\left[\delta_{i k}-\frac{u\left(x_{i}\right)}{u\left(x_{k}\right)} \lambda_{k}\left(x_{i}\right)\right] \alpha_{k}=(g u)\left(x_{i}\right), \quad i=1, \ldots, j .
$$

By solving this system we can find, $\alpha_{i}, i=1, \ldots, j$ and so the approximate solution of integral equation will be obtained.

\section{Numerical Examples}

Example 1. Consider the equation

$$
f(y)-\int_{0}^{+\infty} x y f(x) e^{\frac{-x}{2}} d x=e^{-y}-0.444444 y,
$$

with $k(x, y)=x y$ and $u(x)=e^{\frac{-x}{2}}$ and $\theta=0.5$ and consider the equation in $C_{u}$, with exact solution $f(x)=e^{-x}$. We can construct and solve the system (5). In Table 1, we report the values of the weighted error $u(y)\left|e^{-y}-\bar{f}_{n}(y)\right|$ at some points.

Example 2. Let

$$
f(y)-\int_{0}^{+\infty} 2 x y f(x) e^{-x} d x=e^{-y}-0.5 y
$$

Here $k(x, y)=2 x y$ and $w(x)=e^{-x}$. Taking $u(x)=e^{\frac{-x}{2}}$ and $\theta=0.5$, we can construct and solve the system (7). In Table 2, we report the values of the weighted error $u(y)\left|e^{-y}-\bar{f}_{n}(y)\right|$ at some points. 


\begin{tabular}{|l|l|l|l|l|l|}
\hline$n$ & $y=1$ & $y=10$ & $y=100$ & $y=1000$ & $y=10000$ \\
\hline 16 & $2.17603(-1)$ & $5.35497(-4)$ & $2.831(-23)$ & $3.97283(-216)$ & $2.057608(-2168)$ \\
32 & $1.43843(-1)$ & $5.11362(-4)$ & $1.80273(-25)$ & $1.07388(-213)$ & $7.800829(-2163)$ \\
64 & $2.2313(-1)$ & $3.05902(-7)$ & $5.27528(-29)$ & $7.80687(-235)$ & $8.9784356(-2199)$ \\
\hline
\end{tabular}

Table 1: Weighted errors $\left|\left[e^{-y}-\bar{f}_{n}(y)\right] u(y)\right|$ at the points $y=$ 1, 10, 100, 1000, 10000. for Example 1.

\begin{tabular}{|l|l|l|l|l|l|}
\hline$n$ & $y=1$ & $y=10$ & $y=100$ & $y=1000$ & $y=10000$ \\
\hline 16 & $7.187331(-2)$ & $3.01172(-4)$ & $1.59291(-23)$ & $2.23538(-216)$ & $3.01172(-2168)$ \\
32 & $6.31523(-2)$ & $1.03209(-3)$ & $3.6374(-25)$ & $2.16679(-213)$ & $3.081485(-2161)$ \\
64 & $2.2313(-1)$ & $3.05901(-7)$ & $1.05438(-28)$ & $1.56023(-234)$ & $1.794537(-2198)$ \\
\hline
\end{tabular}

Table 2: Weighted errors $\left|\left[e^{-y}-\bar{f}_{n}(y)\right] u(y)\right|$ at the points $y=$ 1, 10, 100, 1000, 10000. for Example 2.

\begin{tabular}{|l|l|l|l|l|l|}
\hline $\mathrm{n}$ & $\mathrm{y}=1$ & $\mathrm{y}=10$ & $\mathrm{y}=100$ & $\mathrm{y}=1000$ & $\mathrm{y}=10000$ \\
\hline 16 & $7.09255(-2)$ & $7.67593(-14)$ & $1.01124(-222)$ & $7.2671(-3216)$ & $1.75964(-42170)$ \\
32 & $6.98888(-2)$ & $1.68034(-4)$ & $3.83948(-29)$ & $1.40611(-217)$ & $9.183649(-2169)$ \\
64 & $1.11565(-1)$ & $1.17938(-17)$ & $3.36694(-229)$ & $1.040014(-3235)$ & $9.12940735(-42201)$ \\
\hline
\end{tabular}

Table 3: Weighted errors $\left|\left[e^{-y}-\bar{f}_{n}(y)\right] u(y)\right|$ at the points $y=$ 1, 10, 100, 1000, 10000. for example 3 .

Example 3. Here we consider the equation

$$
f(y)-\int_{0}^{+\infty} 2 x y^{3} f(x) x e^{-x} d x=e^{-y}-1.18519 y^{3}
$$

with $k(x, y)=2 x y^{3}$ and $w(x)=x e^{-x}$. Taking $u(x)=x^{\frac{1}{2}}(1+x)^{-1} e^{\frac{-x}{2}}$ and $\theta=0.5$, we obtain the results presented in Table 3 .

Example 4. Consider the equation

$f(y)-\int_{0}^{+\infty}(2 x+y) e^{-x+y} f(x) x^{4} e^{-x} d x=e^{y}(-2.02752-0.94464 y)+3 \sin (x)$.

Where $k(x, y)=(2 x+y) e^{-x+y}$ and $w(x)=x^{4} e^{-x}$ with the exact solution $f(x)=3 \sin (x)$. Taking $u(x)=x^{2}\left(\frac{x}{1+x}\right)(1+x)^{-2} e^{\frac{-x}{2}}$ and $\theta=0.4$, we obtain the results presented in Table 4 . 


\begin{tabular}{|l|l|l|l|l|l|}
\hline$n$ & $y=1$ & $y=10$ & $y=100$ & $y=1000$ & $y=10000$ \\
\hline 16 & $2.13305(-2)$ & $3.91871(-2)$ & $6.0038(-21)$ & $1.13907(-213)$ & $1.13931(-213)$ \\
32 & $8.19234(-2)$ & $8.28765(-3)$ & $2.98861(-22)$ & $1.00302(-209)$ & $7.556268(-2158)$ \\
64 & $8.61788(-2)$ & $3.96789(-2)$ & $1.87203(-22)$ & $3.22162(-214)$ & $3.815379(-2172)$ \\
\hline
\end{tabular}

Table 4: Weighted errors $\left|\left[3 \sin (x)-\bar{f}_{n}(y)\right] u(y)\right|$ at the points $y=$ 1, 10, 100, 1000, 10000. for example 4 .

\section{References}

[1] K.E. Atkinson, The Numerical Solution of Integral Equations of the Second Kind, Cambridge University Press, Cambridge (1997).

[2] M.C. De Bonis, B. Della Vecchia, G. Mastroianni, Approximation of the Hilbert transform on the real semiaxis using Laguerre zeros, Journal of Computational and Applied Mathematics, 140 (2002), 209-229.

[3] G. Mastroianni, D. Occorsio, Some quadrature formulae with nonstandard weights, SIAM Journal on Numerical Analysis, 235 (2010), 602-614.

[4] L. Fermo, A Nyström method for a class of Fredholm integral equations of the third kind on unbounded domains, Applied Numerical Mathematics, 59 (2009), 2970-2989.

[5] D. Occorsio, A method to evaluate the Hilbert transform on $(0,+\infty), A p$ plied Mathematics and Computation, 217 (2011), 5667-5679.

[6] D. Occorsio, Extended Lagrange interpolation in weighted uniform norm, Applied Mathematics and Computation, 211 (2009), 10-22.

[7] M.C. De Bonis, M.G. Mastroianni, Nyström method for systems of integral equations on the real semiaxis, IMA Journal of Numerical Analysis, 29 (2009), 632-650.

[8] G. Mastroianni, G.V. Milovanovic, Some numerical methods for secondkind Fredholm integral equations on the real semiaxis, IMA Journal of Numerical Analysis, 29 (2009), 1046-1066.

[9] G. Mastroianni, G. Monegato, Truncated quadrature rules over $(0,+\infty)$ and Nyström type methods, SIAM J. Numer. Anal., 41 (2003), 1870-1892.

[10] B. Della Vecchia, G. Mastroianni, Gaussian rules on unbounded intervals, in: Numerical Integration and its Complexity (Oberwolfach, 2001), J. Complexity, 19(3), (2003), 247-258. 
[11] G. Mastroianni, D. Occorsio, Markov-Sonin Gaussian rule for singular functions, J. Comput. Appl. Math., 169 (1)(2004), 197-212.

[12] A.S. Cvetkovic, G.V. Milovanovic, The mathematica package "OrthogonalPolynomials", Facta Unive. Ser. Math. Inform., 19 (2004), 17-36.

[13] G. szegö, Orthogonal Polynomials, Amer. Math. Soc. Colloq. Publ., 23, AMS, Providence, RI (1975). 